\title{
Commentary on Kate E. Lynch, Emily C. Parke, and Maureen A. O'Malley: 'How Causal are Microbiomes? A Comparison with the Helicobacter pylori Explanation of Ulcers'
}

\section{Donald Gillies ${ }^{1}$}

Received: 15 July 2019 / Accepted: 3 October 2019 / Published online: 18 November 2019

(C) The Author(s) 2019

\begin{abstract}
This commentary focuses on the authors' treatment of Koch's postulates. It argues in favour of a modification of Koch's postulates and their analysis in terms of necessary and sufficient conditions. This leads to a criticism of the authors' treatment of the C. difficile case, and to query the need for the criteria of specificity and proportionality.
\end{abstract}

Keywords Microbiomes $\cdot$ Causality $\cdot$ Koch's postulates $\cdot$ Helicobacter pylori $\cdot$ C. difficile

Let me begin by saying that there is a great deal in this paper with which I agree, especially the central parts of the paper (pp. 10-15) criticizing current attempts to explain obesity and some mental health conditions in terms of microbiomes. My commentary then will be mainly focussed on those parts of the paper which deal with Koch's postulates and related issues.

The authors discuss Koch's postulates in connection with the example of whether Helicobacter pylori is the cause of peptic ulcers. They point out, quite correctly, that Koch's postulates could not be fully satisfied in this case because of the absence of an animal model, which made the case very similar to that of cholera. My own view is that the possible absence of an animal model makes it desirable to modify Koch's postulates (see Gillies 2019, Section 3.4, pp. 55-57). My version of Koch's postulates differs from that of Lynch et al., but the point is easily stated in terms of their formulation. Their third postulate is (p.3):

This comment refers to the article available at (https://doi.org/10.1007/s10539-019-9702-2).

Donald Gillies

donald.gillies@ucl.ac.uk

1 Department of Science and Technology Studies, University College London, Gower Street, London WC1E 6BT, UK 
3. After being fully isolated and repeatedly grown in pure culture, the microorganism can induce the disease by being introduced into a healthy animal host.

Now this is a productive action based on causality, that is to say an action which produces the disease. However, for medicine, it is much more important to have avoidance actions based on causality, that is to say actions which prevent or cure the disease rather than producing it. Koch's mistake, on this account, was to have a postulate corresponding to productive actions, but not one corresponding to the more crucial avoidance actions. This is easily remedied by replacing postulate 3 by the postulate either $3 \mathrm{a}$ or $3 \mathrm{~b}$, where $3 \mathrm{a}$ is the old 3 , and $3 \mathrm{~b}$ is

3b. If the microorganisms are prevented from multiplying in the patient's body, then the patient will not have the disease.

Both the cholera and the helicobacter pylori cases do satisfy Koch's postulates modified in this way. This modification also has the advantage that it makes the kind of risky self-experimentation carried out by Marshall quite unnecessary.

Lynch et al. say (p. 7, Footnote 5):

These issues point to a possible interpretation of Koch's postulates as being about necessary and sufficient conditions. While they are sometimes interpreted this way (e.g., Susser 1991), this is not what is claimed under an interventionist account of the postulates (Ross and Woodward 2016).

Actually I do support analysing Koch's postulates in terms of necessary and sufficient conditions. Consider a causal law of the form A causes D where D is a disease. We can base a productive action on this law if we can instantiate A and A is a sufficient condition for D. This corresponds to $3 \mathrm{a}$ in the modified Koch's postulates. We can base an avoidance action on this law if we can instantiate not-A, and A is a necessary condition for D. This corresponds to $3 \mathrm{~b}$ in the modified Koch's postulates. The best causal laws for medicine are those in which $\mathrm{A}$ is a necessary condition for $\mathrm{D}$ and we can prevent $\mathrm{A}$ from occurring. A recent example is the discovery that a preceding infection by the papilloma virus is a necessary condition for cervical cancer to develop. This means that cervical cancer can be prevented by vaccinating against the papilloma virus.

This point of view leads to a criticism of some aspects of the authors' treatment of the clostridium difficile case (pp. 15-16). It seems that an infection by clostridium difficile is associated with an unhealthy microbiome. If the unhealthy microbiome of the patient is replaced by a healthy microbiome using the technique of FMT (faecal microbiome transplant), then the clostridium difficile infection disappears, or at least is considerably ameliorated. On the basis of this evidence, it seems to me that we can accept the causal law:

An unhealthy microbiome causes clostridium difficile infections (*)

where the cause here is understood as a necessary, but not sufficient, condition for the effect. Moreover (*) is a causal law of just the kind which is most useful for medicine. 
The law (*) seems to me therefore a success for the microbiome research programme, which consequently has something to its credit even though its attempts to explain obesity and some mental health conditions have been failures. However, Lynch et al. comment on the situation as follows (p. 16):

The success has been interpreted as evidence in favour of the whole microbiome being causally efficacious in curing $C$. difficile. But does this treatment indicate specificity and proportionality?

This example seems to me to show that the criteria of specificity and proportionality do not need to be satisfied to establish satisfactory causal laws in medicine. Of course these criteria might be useful heuristics for further research in the field. For example, if we could replace $(*)$ by a more specific causal law, this might lead to a treatment which could be easier, cheaper, less invasive, etc. than FMT. However, such a development would not show that $(*)$ is invalid, or that it is wrong to regard the whole microbiome as being causally efficacious.

Open Access This article is distributed under the terms of the Creative Commons Attribution 4.0 International License (http://creativecommons.org/licenses/by/4.0/), which permits unrestricted use, distribution, and reproduction in any medium, provided you give appropriate credit to the original author(s) and the source, provide a link to the Creative Commons license, and indicate if changes were made.

\section{Reference}

Gillies D (2019) Causality, probability, and medicine. Routledge, London

Ross LN, Woodward JF (2016) Koch's postulates: an interventionist perspective. Stud Hist Philos Biol Biomed Sci 59:35-46

Susser M (1991) What is a cause and how do we know one? A grammar for pragmatic epidemiology. Am J Epidemiol 133(7):635-648

Publisher's Note Springer Nature remains neutral with regard to jurisdictional claims in published maps and institutional affiliations. 